\title{
DOJON: VIRTUALIZANDO O CONCEITO DO CODING DOJO EM UM AMBIENTE COLABORATIVO PARA A PRÁTICA DE PROGRAMAÇÃO
}

\author{
Sahudy Montenegro González ${ }^{1}$, Annabell del Real Tamariz ${ }^{2}$ e Natalie Fernández del Real ${ }^{3}$ \\ ${ }^{1}$ Universidade Federal de São Carlos, Departamento de Computação de Sorocaba, Rod. SP-264, KM 110. Sorocaba, \\ SP, 18052-780, Brasil \\ ${ }^{2}$ Universidade Estadual do Norte Fluminense, Laboratório de Ciências Matemáticas, Brasil \\ ${ }^{3}$ Universidade Estadual do Norte Fluminense, Av. Alberto Lamego, 2000. Campos dos Goytacazes, RJ,
} 28013-602, Brasil

\begin{abstract}
RESUMO
Um Coding Dojo é um encontro de programadores para trabalhar em conjunto desafios de programação. O objetivo é que os participantes aprendam e treinem de uma maneira divertida, melhorando suas habilidades de programação e de trabalho em grupo. Com o intuito de melhorar a aprendizagem, propõe-se a virtualização do conceito de Coding Dojo. Este artigo apresenta o sistema DojOn (Dojo Online), um ambiente Web colaborativo para a prática de programação. Para atender os requisitos do Coding Dojo, o ambiente é composto por: (1) um editor de texto; (2) compilador/interpretador para várias linguagens; (3) chat para a interação entre usuários; (4) um gerenciador de tempo; e (5) um repositório de problemas. Adicionalmente, apresenta-se uma proposta de avaliação dos resultados de uso do ambiente, que visa medir com estatística descritiva a interdependência entre a aprendizagem colaborativa e a aprendizagem individual, a partir da análise das interações dos usuários no ambiente.
\end{abstract}

\section{PALAVRAS-CHAVE}

Aprendizagem Colaborativa, Coding Dojo, Programação em Pares, Ambiente Virtual Colaborativo, Estatística Descritiva, Matriz de Adjacência

\section{INTRODUÇÃO}

O aprendizado colaborativo se baseia em princípios construtivistas. Espera-se que os aprendizes construam conhecimento mediante interação e colaboração em pequenos grupos, resolvendo um desafio em comum e evitando a competitividade (Nyikos \& Hashimoto, 1997). O Conselho Nacional de Pesquisa dos Estados Unidos (Pellegrino \& Hilton, 2012) recomendou que as instituições de ensino superior desenvolvessem as habilidades de colaboração e trabalho em equipe em seus alunos, como parte das chamadas competências do Século XXI. Adicionalmente, trabalhos científicos mostram evidências de que a interação social em ambientes virtuais contribui com o aprendizado efetivo (Hiltz, et al., 2000). De acordo com (Prikladnicki, 2015), alguns estudos da literatura relatam que a programação em grupo, como por exemplo Coding Dojo, apresenta bons resultados no ensino de programação.

Um Coding Dojo (Sato, et al., 2008) é um encontro onde um grupo de programadores se reúne para trabalhar concomitantemente em um desafio de programação. O objetivo é que os participantes aprendam e treinem de uma maneira divertida, através de uma metodologia pragmática, melhorando, assim, suas habilidades de programação e de trabalho em grupo. Como parte do trabalho descrito em (Da Luz, et al., 2013), uma pesquisa digital com diferentes grupos de Coding Dojo no Brasil foi realizada, e concluiu-se que essa atividade favorece a colaboração em ambientes de aprendizado. Em (Heinonen, et al., 2013), expõe-se que $82 \%$ dos participantes (alunos de cursos de Engenharia de Software) recomendariam a participação em sessões de Coding Dojo para seus colegas.

Desta forma, apresentam-se algumas evidências de que um ambiente colaborativo integrado à metodologia do Coding Dojo pode ser de benefício para a aprendizagem de programação ou o reforço dessa aprendizagem. Assim, o objetivo deste artigo é apresentar o ambiente virtual colaborativo, DojOn - Dojo 
Online, que visa propiciar o encontro entre usuários para a prática de programação, seguindo a metodologia do Coding Dojo, com a finalidade de facilitar sua realização, usufruindo dos vários benefícios que a Web oferece. Para isso, o sistema visa proporcionar os recursos e requisitos do Coding Dojo em sessões online.

Para a melhor compreensão do trabalho, este é dividido da seguinte maneira: a Seção 2 oferece uma visão geral da metodologia do Coding Dojo; a Seção 3 apresenta a justificativa da relevância da proposta a partir de trabalhos relacionados; a Seção 4 descreve o sistema DojOn e expõe os princípios de projeto básicos, sua arquitetura, o protótipo e um cenário que ilustra seu funcionamento; a Seção 5 propõe um método de avaliação dos resultados de uso do ambiente por meio das interações dos usuários; por fim, as considerações finais do trabalho e as perspectivas de trabalhos futuros são apresentadas na Seção 6.

\section{VISÃO GERAL DO CODING DOJO}

Coding Dojo (Sato, et al., 2008) (Bravo \& Goldman, 2010) é uma atividade que tem sido utilizada por programadores para treinar práticas ágeis de programação. Em uma sessão de Coding Dojo, programadores se juntam para aprender uns com os outros em um ambiente social amigável. O objetivo do Coding Dojo é a prática de metodologias ágeis, especialmente, o desenvolvimento guiado por testes (Test Drive-Development - TDD), programação em pares e baby steps para o aprendizado, aperfeiçoamento e, até desenvolvimento de sistemas. O propósito principal é que o participante melhore a forma como escreve seus códigos de programas, podendo aprender com a experiência de outros participantes. Isto pode ser corroborado com o trabalho experimental descrito em (Da Luz, et al., 2013), o qual teve 64 participantes (com mais de 10 sessões de Coding Dojo). A maioria dos participantes da pesquisa (64\%) respondeu que os baby steps simplificam o processo de achar a solução, o uso de TDD ajuda aprender a metodologia e a programação em par ajuda a nivelar o grupo.

Um Coding Dojo normalmente é realizado em um intervalo de tempo de uma a duas horas. Ele pode ser realizado em diversas linguagens de programação. O primeiro passo para realizar um Coding Dojo é escolher um desafio de programação. Não há a necessidade de resolver ou terminar o desafio durante o Coding Dojo. Em turnos de tempo limitado, um par de programadores 〈piloto e copiloto > trabalha na solução, usando um computador, fazendo pequenos passos na direção da solução, usando TDD e, o mais importante, comunicando a ideia aos espectadores. A tela desse computador é projetada para que todos os participantes tenham acesso ao que está sendo desenvolvido pela dupla. Quando o tempo acaba, o piloto, que é o que está escrevendo o código, se junta ao resto dos espectadores. O copiloto, que é quem ajuda o piloto, passa para o lugar do piloto, sendo a vez de ele escrever código. Um voluntário da plateia torna-se copiloto. Cada turno pode ter um programador mais experiente e um iniciante, para assim aprenderem juntos.

\section{JUSTIFICATIVA E TRABALHOS RELACIONADOS}

Alguns trabalhos foram conduzidos com o fim de investigar os benefícios que o Coding Dojo pode trazer aos estudantes de programação e ao desenvolvimento de software, tais como (Oliveira, et al., 2018) (Heinonen, et al., 2013) (Da Luz, et al., 2013) (Rocha, et al., 2018) (Fonseca, et al., 2019). Em todos os casos, as conclusões são claras e afirmam que os alunos envolvidos nos experimentos executados alegam que a experiência é positiva.

Por outro lado, pesquisas recentes apostam em ambientes virtuais colaborativos em diversas áreas e destacam os benefícios do uso desses ambientes para o desenvolvimento promissor dos seus usuários. Dentre elas, os autores de (Jevremovic, et al., 2018) propõe uma ferramenta virtual, WIDE, para uso colaborativo na execução de atividades para o ensino da disciplina de Desenvolvimento Web. ZHANG, et al. (2018) descrevem o ambiente CoMove para ajudar na interação social e comunicação de pacientes com Autism Spectrum Disorder (ASD).

A proposta de um ambiente como o DojOn pode tirar proveito das vantagens da tecnologia de ambientes virtuais aliada à metodologia do Coding Dojo. Para reforçar esta proposta, no contexto de um estudo de caso o autor da pesquisa descrita em (Estácio, 2017), que estava presente em sessões de Coding Dojo, identificou melhorias a partir de comentários dos participantes e o autor afirma que "uma infraestrutura adequada se faz importante para a dinâmica da prática, especialmente um conjunto de atalhos que seja entendível para todos 
os participantes"(p.111), o que seria simples de suprir quando os alunos podem trabalhar no seu próprio computador de maneira online. Este é o caso dos Virtual Pair Programming (VPP). O trabalho descrito em (Zacharis, 2010) (Zacharis, 2009) expõe experimentos de ensino de Java usando VPP, com resultados que confirmam as hipóteses que estudantes em pares programam usando um código melhor, e percebem a experiência de aprendizado virtual e em pares como sendo positiva.

\section{DOJON}

Os usuários indicados para usar o DojOn são programadores ou iniciantes em programação. Em uma mesma sessão, podem haver usuários com diferentes níveis de experiência, o que é recomendado, pois a situação acaba gerando aprendizado para aqueles mais novatos e desafios para aqueles mais experientes e entendimento mútuo do problema (Nyikos \& Hashimoto, 1997) (França \& Tedesco, 2014). Uma vez começada uma sessão, cria-se uma fila com o primeiro participante que começará o desafio e a sequência dos próximos. Um problema de programação é exibido para que todos o visualizem. Define-se, então, um valor de tempo ( $n$ minutos) para todo participante acatar na sua vez. Após $n$ minutos, é a vez do próximo participante na sequência. Essa rotina acontece até o problema ser resolvido ou até que não haja mais participantes na sessão. Todos podem se comunicar via chat. A Figura 1 é o fluxograma do sistema, que ilustra o processo descrito anteriormente.

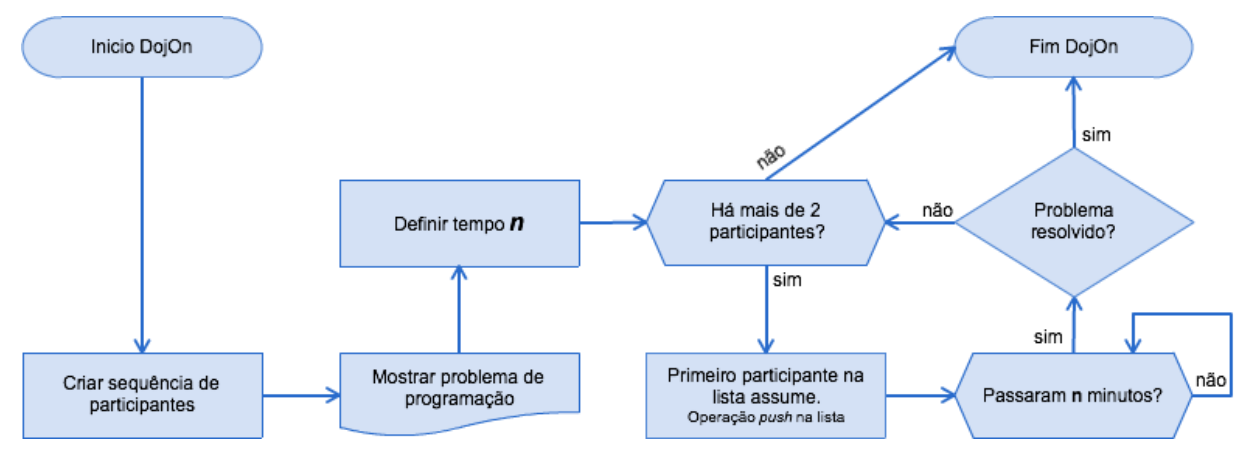

Figura 1. Fluxograma do DojOn

Com o uso de TDD, os participantes do Coding Dojo, além de escrever código em uma linguagem de programação, devem escrever testes, constituindo o único mecanismo de verificação do funcionamento correto do código. Existem variados frameworks para suporte de testes automatizados. Normalmente, cada framework permite testar uma linguagem de programação específica, por exemplo: RSpec permite testar código em Ruby, JUnit em Java, Jasmine em JavaScript.

\subsection{Objetivos do DojOn}

Assim como exposto em (França \& Tedesco, 2014), com a utilização contínua do sistema, espera-se desenvolver habilidades nos participantes. Elas são: (1) trabalhar de maneira colaborativa para produzir uma solução de consenso; (2) discutir alternativas de soluções de problemas durante o desenvolvimento da solução de consenso. Consequente e inerentemente ao processo, espera-se dos participantes: (3) o desenvolvimento do pensamento computacional; (4) aprender a partir do conhecimento dos outros participantes; (5) desenvolver o próprio conhecimento a partir da revisão das soluções; e (6) auto aprimorar o processo de resolução de problemas de programação a partir do feedback fornecido pelos pares, pois durante as sessões se apontam equívocos e sugerem-se melhorias das soluções do problema tratado. 


\subsection{Requisitos do DojOn}

Para funcionar de acordo com o previsto, o sistema deve atender dez requisitos. Os mesmos foram divididos em dois conjuntos. O primeiro refere-se aos requisitos impostos pelo Coding Dojo ou os recursos para agir de acordo com sua metodologia. O segundo conjunto se refere aos princípios de projetos definidos pelas pesquisadoras, autoras deste trabalho.

Do Coding Dojo: (1) ser colaborativo: permitir que a solução de um problema seja construída em conjunto; (2) permitir interação comunicativa entre os usuários; (3) ter um gerenciador de tempo de uso dos participantes; (4) apresentar um problema de programação; (5) permitir desenvolvimento guiado a testes; (6) para cumprir o anterior, o editor de código deve permitir a criação/edição de, pelo menos, dois arquivos, um para o código e outro para teste; e (7) começar as sessões de Coding Dojo com a presença virtual de três ou mais participantes. No caso deste último requisito, em uma sessão de Coding Dojo presencial, os usuários devem esperar que haja pelo menos dois participantes. Porém, a recomendação do autor de (Estácio, 2017) é a presença de três participantes, no mínimo. Com isto, esperam-se resultados mais satisfatórios quanto à aprendizagem colaborativa.

De sistema: (8) ser Web-IDE; (9) ser de código aberto; e (10) oferecer suporte a várias linguagens de programação e não se restringir a apenas uma, assim como, oferecer suporte para a inclusão de novas linguagens de programação.

\subsection{Módulos do Sistema}

Para atender os requisitos listados na Seção 4.2, o sistema possui os seguintes componentes básicos: editor de texto; compilador e/ou interpretador; meio de comunicação (chat) entre os participantes da sessão; gerenciador de uso; gerenciador de tempo; e gerenciador de problemas de programação.

A Figura 2 mostra a estrutura de DojOn e a relação entre os componentes básicos do sistema. O usuário pode: digitar o código fonte do programa no editor de texto; solicitar a execução de um arquivo no compilador/interpretador (que retorna mensagens de erros e/ou a saída do arquivo); interagir com outros usuários e ter retorno mediante o meio de comunicação; escolher o intervalo de tempo para utilização do editor, usando o gerenciador de tempo (que mostra o tempo restante que o usuário ainda tem); ler e mudar de problema de programação, usando o gerenciador de problemas.

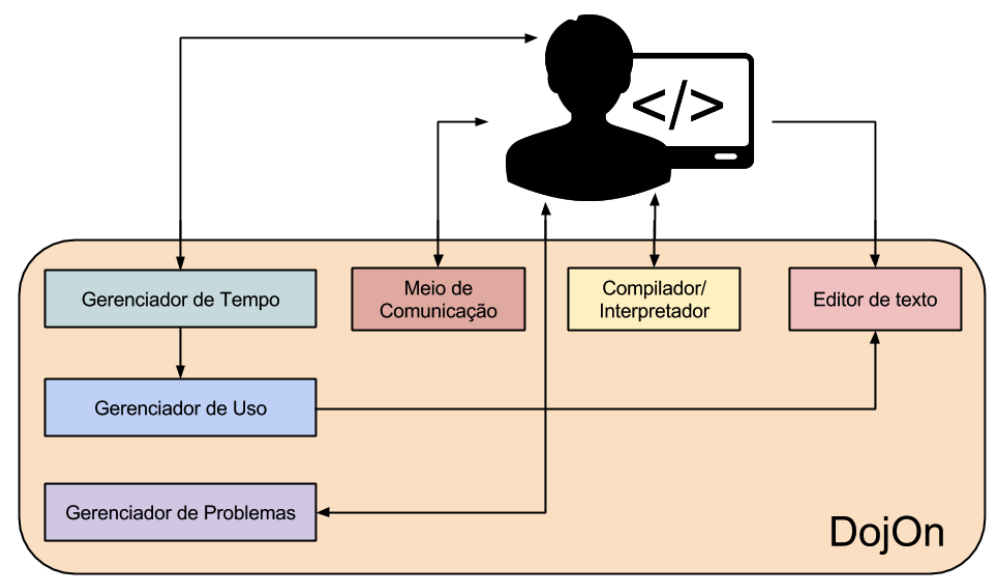

Figura 2. Módulos do DojOn

O DojOn tira proveito dos recursos já disponíveis no Cloud9, integrando os mesmos como parte do ambiente. Adicionalmente, o DojOn é encarregado de desenvolver os gerenciadores de tempo, de usuários e de problemas de programação, que são recursos referentes ao Coding Dojo. O Dojo Puzzles é uma página Web que possui um repositório de problemas de programação. O gerenciador de problemas do DojOn mostra na interface, para os usuários, problemas de programação presentes no Dojo Puzzles, simplificando o desenvolvimento do módulo ao reusar uma base de problemas. 
O gerenciador de tempo mostra um relógio com a contagem regressiva do tempo restante, além de emitir um aviso ao acabar. Esse gerenciador repassa para o gerenciador de uso o intervalo de tempo escolhido pelo usuário. O gerenciador de uso mantém uma fila de participantes por sessão e habilita ou desabilita a utilização do editor, mostrando um sinal vermelho ou verde para avisar ao usuário que é ou não a sua vez. Portanto, os recursos do DojOn são quatro: o timer, o sinal, o problema e o chat. A pesar do chat não ser um recurso especificamente manipulado por nenhum dos gerenciadores, ele é um recurso que o ambiente disponibiliza para interação entre os usuários.

A Tabela 1 mostra um resumo da execução dos requisitos de projeto, apontando a tecnologia utilizada. Como pode ser observado na tabela, todos os dez requisitos são atendidos.

Tabela 1. Requisitos do DojOn e as tecnologias utilizadas

\begin{tabular}{||l|l||}
\hline \hline Requisitos & Solução \\
\hline \hline $1,2,5,6,8,9$ & DojOn e Cloud9 \\
\hline 3,7 & DojOn \\
\hline 4 & DojOn com Dojo Puzzles \\
\hline 10 & DojOn e Cloud9 (Java, C, Ruby, JavaScript, etc.) \\
\hline \hline
\end{tabular}

\subsection{Cenário de Uso: Rotina de Participação}

Um cenário de uso do sistema é ilustrado na Figura 3. O cenário descreve um desafio em que os participantes terão que implementar uma solução para o problema das Palavras Primas (visualizado no canto esquerdo inferior da tela). Este cenário visa descrever o processo de participação de um usuário no DojOn, detalhando-se a sequência de passos a ser seguida por este e pelos demais participantes. No mesmo, mostram-se os recursos e os gerenciadores envolvidos.

Recursos envolvidos: timer, sinal. Gerenciadores envolvidos: de tempo, de uso.

Quando um usuário decide começar, este deve apertar o botão Começar e um sinal verde será acesso (destacados em vermelho na Figura 3). O tempo predefinido começará a contar (neste caso, de cinco minutos). Assim que o tempo acabar, aparece uma mensagem informando que o tempo se esgotou, e um sinal vermelho será acesso. É a vez de outro usuário. Este agora deve apertar o botão Começar novamente. A contagem do tempo inicia-se mais uma vez (mais cinco minutos). Essa rotina se repete até que o problema seja terminado. Aproveitando a figura, a interface mostra, também, dois participantes da sessão interagindo no chat.

\section{AVALIAÇÃO DOS RESULTADOS}

A aprendizagem colaborativa pressupõe a realização de atividades de forma coletiva, onde, a tarefa de um complementa o trabalho de outros (Silveira, et al., 2012), (Silva, et al., 2015), que é a proposta do Coding Dojo. Partindo dessa afirmação, a hipótese criada é: a colaboração entre usuários com níveis diferentes de experiência influencia na finalização do problema? A partir da hipótese criada, espera-se analisar se os objetivos do sistema estão sendo atingidos. Os indicadores definidos para a avaliação são: a tendência na formação de pares em uma sessão e a correlação desse primeiro indicador com a finalização do problema. Sato et al. (2008) esclarecem que o objetivo do Coding Dojo não é finalizar a tarefa, mas contribuir com a aprendizagem. Porém, a finalização do problema é uma consequência do aprendizado. A finalização do problema envolve a coleta de: (i) o tempo de resolução do mesmo, e (ii) seu sucesso (0: não chegou em uma solução, 1: problema resolvido com sucesso).

Para avaliar a interdependência entre a aprendizagem colaborativa e a aprendizagem individual, utiliza-se a análise das interações dos usuários no ambiente. Para isto, coletam-se dados através do sistema e da observação dos participantes em sessões on-line. Em (Silva, et al., 2015), um grafo direcionado e a matriz de adjacência correspondente são utilizados para representar e analisar as conversações numa sala de bate-papo mediante a associação de cada mensagem enviada. Neste trabalho, usa-se um grafo direcionado para representar a interação dos pares <piloto,copiloto> para cada sessão do DojOn. Para facilitar a observação e a coleta de dados, a resolução de cada problema é considerada uma sessão. 


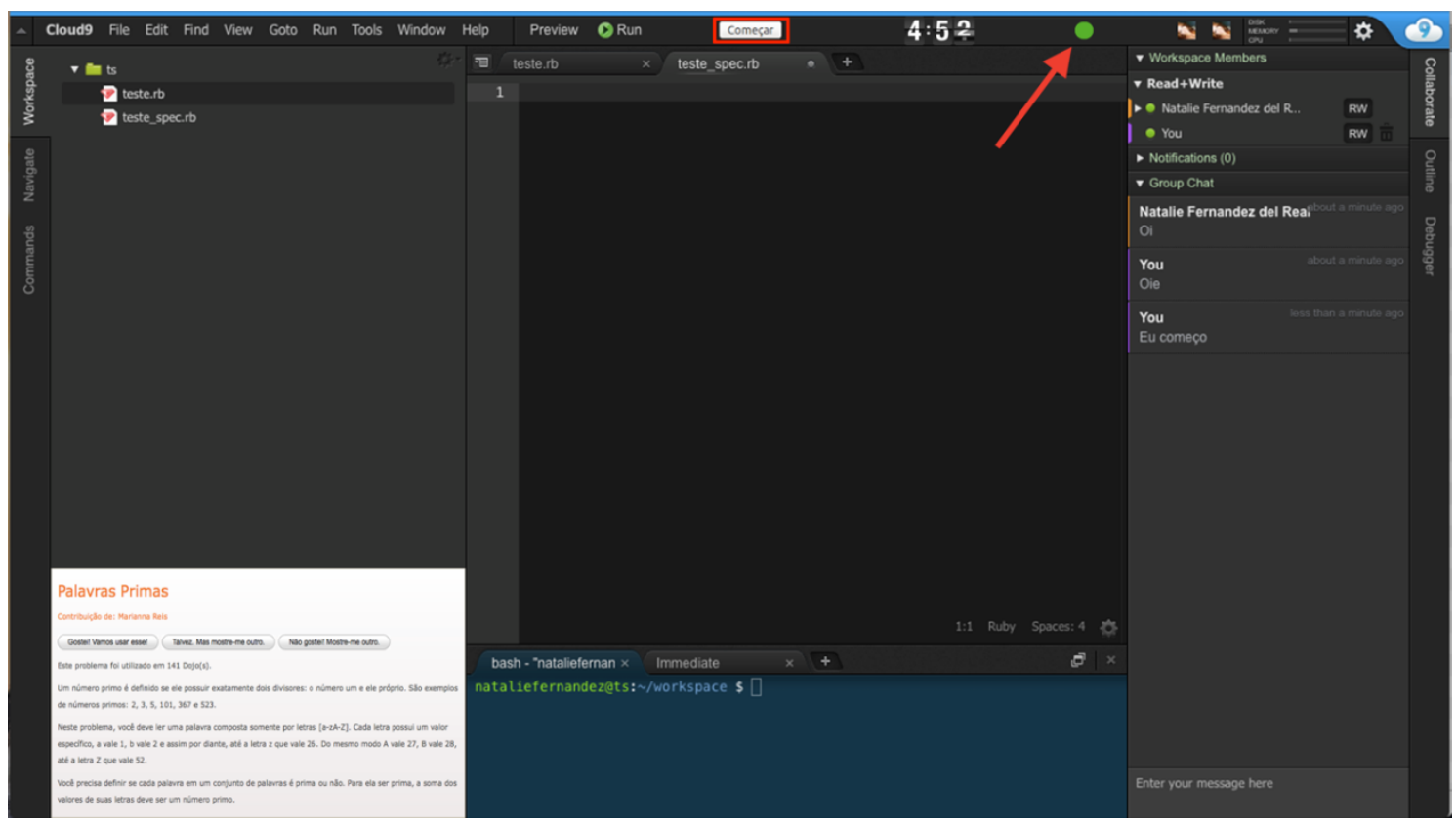

Figura 3. Interface do sistema: Usuário clica em Começar, a contagem regressiva começa e um sinal verde aparece

Para cada sessão, observa-se, também, o tempo de duração estabelecido para cada par de usuários, o nível de dificuldade do problema e se uma solução para o problema foi atingida. A primeira característica é coletada pelo sistema e as últimas duas características são coletadas por observação no experimento.

A interação entre participantes de uma sessão pode ser representada por um grafo direcionado e rotulado, $G=\{U, A\}$, onde $U$ é o conjunto dos usuários participantes da sessão de DojOn e $A$ é conjunto de pares ordenados $\left\langle U_{i}, U_{j}\right\rangle, U_{i}, U_{j} \in U$. As arestas em $A$ representam a participação em pares em uma sessão, onde $U_{i}$ é o piloto e $U_{j}$ é o copiloto. O grafo pode ser desconexo, pois pode existir, pelo menos, um par de usuários que não está ligado por nenhum caminho, caso que pode acontecer quando um copiloto não quer assumir a posição de piloto na sua vez (na Figura 4(a), o par 〈Enrico,Luis〉).

Um grafo pode ser expressado por uma matriz de adjacência $M$, onde cada entrada $M_{i, j}$ contém o somatório dos pesos da experiência dos usuários envolvidos $U_{i}$ e $U_{j}$. Para isto, cada usuário do sistema possui a propriedade experiência em programação. Essa propriedade pode ter dois valores: peso 0,5 para nada ou pouco experiente, peso 1,0 para programadores com experiência. Ao entrar no sistema, cada usuário é obrigado a configurar esta característica. Assim, os possíveis valores para as células de $M$ são $O$ (sem interação), 1 (piloto e copiloto inexperientes), 1,5 (piloto e copiloto com diferentes níveis de experiência) e 2 (piloto e copiloto experientes).

Usando a matriz de adjacência $M$ de $G$, o grau de saída $S_{i}$ de um vértice $i$ representa quantas vezes o usuário $U_{i}$ participou como piloto na sessão e pode ser obtido contando o número de entradas positivas da linha $i$ de $M$. O grau de entrada $E_{j}$ de um vértice $j$ representa quantas vezes o usuário $U_{j}$ participou como copiloto na sessão e pode ser obtido contando o número de entradas positivas da coluna $j$ de $M$.

Para avaliar se o compartilhamento de experiência entre os participantes de uma sessão influencia na finalização do problema, as métricas propostas para avaliação da hipótese avaliam a distribuição estatística dos pares de participantes. Considera-se como melhores, os valores centrais $(1,5)$, que significam uma distribuição ideal de pares <piloto,copiloto> com diferentes níveis de experiência. Para valores de 1 , a sessão teve mais pares de participantes inexperientes e, para valores de 2, a sessão teve mais pares de experientes.

As métricas são: a moda dos valores positivos da matriz de adjacência, que mede a tendência central dos pares de usuários em relação à experiência (primeiro indicador); e o coeficiente de correlação de Pearson entre a moda e a finalização do problema (tempo e sucesso) para um conjunto de valores de sessões, possibilitando o cálculo do segundo indicador e a verificação da hipótese (nula, positiva, negativa). 


\subsection{Análise de Dados}

Para ilustrar a proposta de avaliação dos resultados, a Figura 4(a) representa uma sessão de Coding Dojo acontecida na universidade para implementar o problema do Jogo de Tênis na linguagem Ruby. Nela, participaram 9 usuários (identificados por pseudônimos). Ao finalizar a sessão, após 90 minutos, o problema não foi resolvido.

(a)

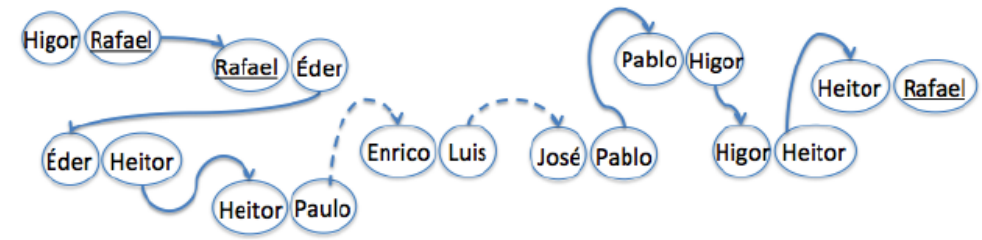

(b)

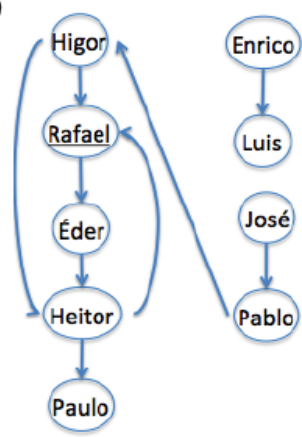

(c)

\begin{tabular}{|c|c|c|c|c|c|c|c|c|c|c|}
\hline & Higor & Rafael & Éder & Heitor & Paulo & Enrico & Luis & José & Pablo & $\begin{array}{c}\text { Grau de } \\
\text { sá́da }\end{array}$ \\
\hline Higor & - & 1.5 & 0 & 1.0 & 0 & 0 & 0 & 0 & 0 & 2 \\
\hline Rafael & 0 & - & 1.5 & 0 & 0 & 0 & 0 & 0 & 0 & 1 \\
\hline Éder & 0 & 0 & - & 1.0 & 0 & 0 & 0 & 0 & 0 & 1 \\
\hline Heitor & 0 & 1.5 & 0 & - & 1.0 & 0 & 0 & 0 & 0 & 2 \\
\hline Paulo & 0 & 0 & 0 & 0 & - & 0 & 0 & 0 & 0 & 0 \\
\hline Enrico & 0 & 0 & 0 & 0 & 0 & - & 1.0 & 0 & 0 & 1 \\
\hline Luis & 0 & 0 & 0 & 0 & 0 & 0 & - & 0 & 0 & 0 \\
\hline José & 0 & 0 & 0 & 0 & 0 & 0 & 0 & - & 1.0 & 1 \\
\hline Pablo & 1.0 & 0 & 0 & 0 & 0 & 0 & 0 & 0 & - & 1 \\
\hline $\begin{array}{l}\text { Grau de } \\
\text { entrada }\end{array}$ & 1 & 2 & 1 & 2 & 1 & 0 & 1 & 0 & 1 & \\
\hline
\end{tabular}

Figura 4. Uma sessão: (a) Sequência completa dos pares 〈piloto,copiloto〉

(b) Grafo da sessão

(c) Matriz de adjacência

O grafo que representa a sessão é ilustrado na Figura 4(b). O Rafael (sublinhado) é o único participante desenvolvedor de aplicações com anos de experiência e os outros 8 participantes são alunos do curso de Ciência da Computação. A matriz de adjacência associada ao grafo da sessão é representada na Figura 4(c). A partir dela, pode se observar que o usuário Higor foi 1 vez copiloto e duas vezes piloto durante a sessão.

Para o caso da Figura 4(c), o valor da moda é 1.0, significando que houve um número predominante de pares de usuários inexperientes na sessão. Para essa sessão, essa predominância pode ter influenciado em que o problema não fosse resolvido. Porém, para analisar essa correlação, precisa-se observar múltiplas sessões do sistema. Esta análise não está sendo incluída, devido à restrição do número de páginas.

\section{CONCLUSÃO}

A virtualização de encontros para prática de programação, no estilo do Coding Dojo, foi a proposta deste trabalho, a qual enxergamos como uma contribuição positiva para a comunidade computacional, pois tudo que incentiva e facilita o aprendizado é vantajoso em qualquer área profissional. O trabalho possui duas contribuições importantes: (1) o ambiente colaborativo para programação adotando a metodologia do Coding Dojo, e (2) a proposta de avaliação que visa analisar com estatística descritiva a interdependência entre a aprendizagem colaborativa e a aprendizagem individual.

O ambiente como um todo supre os dez requisitos de sistema e atende a metodologia do Coding Dojo. Para validação da aprendizagem colaborativa foi proposta uma abordagem baseada nas interações entre os participantes, que permite analisar a distribuição dos pares <piloto,copiloto> com diferentes níveis de experiência.

Como trabalho futuro, planeja-se conduzir um estudo de viabilidade com o objetivo de validar a abordagem proposta de avaliação baseada em interações e avaliar, do ponto de vista dos estudantes, se ao utilizar o ambiente eles gostam da atividade, se melhoram as habilidades de programação e se passam a trabalhar melhor em grupo. Testes de usabilidade do sistema e inspeção de interfaces também estão sendo previstos. 


\section{REFERÊNCIAS}

Bravo, M. \& Goldman, A., 2010. Reinforcing the Learning of Agile Practices Using Coding Dojos. In: Agile Processes in Software Engineering and Extreme Programming. Lecture Notes in Business Information Processing. Springer Berlin Heidelberg.

Da Luz, R., Serra Seca Neto, A. \& Vida Noronha, R., 2013. Teaching TDD, the Coding Dojo Style. Proceedings of IEEE 13th International Conference on Advanced Learning Technologies (ICALT), pp. 371-375.

Estácio, B. J. D. S., 2017. Uma avaliação empírica sobre a aprendizagem colaborativa em Coding Dojo Randori no contexto de desenvolvimento de software. Tese (Doutorado) - Pontifícia Universidade Católica do Rio Grande do Sul.

Fonseca, F. M., Silva, E. B. d. \& Mendonça, D. S., 2019. Designing Dojo: A collaborative method for teaching design patterns. Montreal, Quebec, Canada, 12th International Workshop on Cooperative and Human Aspects of Software Engineering (CHASE '19).

França, R. S. d. \& Tedesco, P. C. d. A. R., 2014. Um modelo colaborativo para a aprendizagem do pensamento computacional aliado à autorregulação. Anais do XXV Simpósio Brasileiro de Informática na Educação, pp. 1133-1142.

Heinonen, K., Hirvikoski, K., Luukkainen, M. \& Vihavainen, A., 2013. Learning Agile Software Engineering Practices Using Coding Dojo. s.l., ACM, pp. 97-102.

Hiltz, S. R. et al., 2000. Measuring the Importance of Collaborative Learning for the Effectiveness of ALN: A Multi-Measure, Multi-Method Approach. Journal of Asynchronous Learning Networks, Volume 4, pp. 103-123.

Jevremovic, A., Ristic, N., Veinovic, M. \& al., e., 2018. WIDE: Centralized and Collaborative Approach to Teaching Web Development. Journal of Internet Technology, 19(4), pp. 1003-1014.

Klasnja-Milicevic, A., Ivanovic, M., Vesin, B. \& Budimac, Z., 2018. Enhancing e-learning systems with personalized recommendation based on collaborative tagging techniques. Applied Intelligence, Volume 48, p. 1519-1535.

Nyikos, M. \& Hashimoto, R., 1997. Constructivist theory applied to collaborative learning in teacher education: In search of ZPD. The Modern Language Journal, 81(4), p. 506-517.

Oliveira, C. M. C. d. et al., 2018. Improving Student's Learning and Cooperation Skills Using Coding Dojos (In the Wild!). San Jose, CA, USA, IEEE Frontiers in Education Conference (FIE).

Pellegrino, J. W. \& Hilton, M. L., 2012. Education for life and work: Developing transferable knowledge and skills in the 21 st century. Washington, DC: Committee on Defining Deeper Learning and 21st Century Skills, National Research Council. Board on Testing and Assessment and Board on Science Education, Division of Behavioral and Social Sciences and Education, The National Academies Press.

Prikladnicki, B. J. d. S. E. a. R., 2015. Distributed Pair Programming: A Systematic Literature Review. Information and Software Technology, Volume 63, pp. 1-10.

Rocha, F. G., Sabino, R. F. \& Rodriguez, G., 2018. Using Dojo as a Pedagogical Practice to Introduce Undergraduate Students to Programming. São Paulo, Brazil, XIII Latin American Conference on Learning Technologies (LACLO).

Sato, D., Corbucci, H. \& Bravo, M., 2008. Coding Dojo: An Environment for Learning and Sharing Agile Practices. Conference on Agile, AGILE'08, pp. 459-464.

Silva, V. C. M. d., Pimentel, M. \& Dias, V. M. F., 2015. Bate-papo Colaborativo ou Centrado no Professor?. Anais do XXVI Simpósio Brasileiro de Informática na Educação (SBIE 2015), pp. 1303-1312.

Silveira, L. H. S. D. et al., 2012. Aprendizagem colaborativa numa perspectiva de educação sem distância. Anais do 180. Congresso Internacional de Educação a Distância (CIAED).

Zacharis, N. Z., 2010. Measuring the Effects of Virtual Pair Programming in an Introductory Programming Java Course. IEEE Transactions on Education, 54(1), pp. 168 - 170.

Zacharis, N., 2009. Evaluating the Effects of Virtual Pair Programming on Students' Achievement and Satisfaction. International Journal of Emerging Technologies in Learning, 4(3), pp. 34-39.

ZHANG, L. et al., 2018. Design and Evaluation of a Collaborative Virtual Environment (CoMove) for Autism Spectrum Disorder Intervention. ACM Transactions on Accessible Computing, 11(2). 